\title{
Electron relaxation in disordered gold films
}

\author{
B. I. Belevtsev*, Yu. F. Komnik, E. Yu. Beliayev \\ B. Verkin Institute for Low Temperature Physics $\&$ Engineering, \\ Kharkov, 310164, Ukraine
}

(February 16, 2018)

\begin{abstract}
The analysis of quantum corrections to magnetoconductivity of thin $\mathrm{Au}$ films responsible for by the effect of weak electron localization has made it possible to determine the temperature dependences of electron phase relaxation time in the temperature range $0.5-50 \mathrm{~K}$ for different degrees of crystal lattice disorder. The disorder was enhanced by irradiating the films in vacuum with $3.5 \mathrm{keV}$ Ar ions. The experimental data clearly demonstrate that the contribution of electron-electron interaction to electron phase relaxation increases with disorder and support the theoretical prediction that the frequency of electron-phonon scattering tends to diminish upon a decrease in electron mean free path. It is found that the spin-orbit scattering rate decreases with disorder. In our opinion, such unusual behavior can take place for thin films at decreasing the electron mean free path provided, that the surface electron scattering contributes significantly to the total spin-orbit scattering. 72.15.Lh; 72.15.Rn; 73.20.Fz; 73.05.-h; 73.50.Jt
\end{abstract}




\section{INTRODUCTION}

The problem of crystal lattice disorder effect upon the processes of inelastic and phase relaxation of electrons in metal systems continues to be topical. Theory the electron-electron interaction increases with disordering and the behavior of temperature dependence of electron-electron relaxation time $\tau_{e e}$ depends on system dimensionality. Thus, for a two-dimensional system $\tau_{e e}^{-1} \propto T$, not to $T^{2}$, as it follows from the Fermi-liquid theory for pure metals. Electron relaxation time due to inelastic electron-phonon collisions, $\tau_{e p}$, in pure metals is characterized by the dependence $\tau_{e p}^{-1} \propto T^{3}$ which is also true for weak disorder ${ }^{3}$ with $q_{T} l \gg 1$ ("pure limit") where $q_{T}$ is the thermal phonon wave vector and $l$ is the electron mean free path. In the case of strong disorder with $q_{T} l \ll 1$ ("dirty limit"), the temperature dependence of $\tau_{e p}^{-1}$ should take the form $\tau_{e p}^{-1} \propto l T^{4}$ as reported in $\mathbb{A}^{\mathrm{B}}$. The increase in exponent on $T$ and the unexpected dependence on $l$ (the frequency of electronphonon scattering decreases with $l$ ) can be attributed to the reduction in the electron-phonon interaction on the crossover to a dirty limit. The processes of inelastic electron scattering by oscillating impurities that result in the dependence $\tau_{e p}^{-1} \propto l^{-1} T^{2}$ are considered in Ref. 0. According to Ref. 国 this contribution makes itself evident under electron scattering by transverse phonons at $q_{T} l>1$. However, as shown in Ref. 6, the contribution of this mechanism is cancelled out in dirty limit and is missing in the pure one $\mathbb{A}$.

Hence the pure-dirty limit transition must involve the change of the dependence $\tau_{e p}^{-1} \propto T^{3}$ to $\tau_{e p}^{-1} \propto l T^{4}$. Therefore, for disordered metals both the behavior of temperature dependence of $\tau_{e p}$ and the effect of electron mean free path on $\tau_{e p}$ are currently central problems.

In disordered metals the interference of electron wave functions at low temperatures results in quantum corrections to conductivity that may be attributed to the effects of weak localization (WL) 1 and electron-electron interaction (EEI) corrections are directly related to the characteristic electron relaxation times and electronelectron interaction constants. This permits the phase relaxation time of electron wave function, $\tau_{\varphi}$, (in essence, the inelastic relaxation time) and the spin relaxation time of electrons due to spin-orbit $\left(\tau_{s o}\right)$ and spin-spin $\left(\tau_{s}\right)$ interaction to be determined from the analysis of the experimental temperature and magnetic field dependences of conductivity, $\sigma(T, H)$.

It was found 12 that the temperature dependence of $\tau_{\varphi}$ determined from the quantum corrections to magnetoconductivity of Au films could be described by the relation $\tau_{\varphi}^{-1} \propto T^{2}$ at $T>4.5 \mathrm{~K}$, the latter being associated with the electron-phonon relaxation processes. More recently, the same result was obtained in Refs. 13 15. According to Ref. 14, the contribution of electron-phonon relaxation separated from $\tau_{\varphi}(T)$ for an Au film of $R_{\square} \approx$ $10^{3} \Omega\left(R_{\square}\right.$ is sheet resistance) could be described by the relation $\tau_{e p}^{-1}=A_{p} T^{2}$, where $A_{p}=$ $1.42 \times 10^{9} \mathrm{~s}^{-1} \mathrm{~K}^{-2}$. However, the overheating experiments at ultralow temperatures $(0.03-$ $1 \mathrm{~K}$ ) described in Ref. 16 for Au films of $R_{\square} \simeq 30-100 \Omega$ resulted in an expression $\tau_{e p}^{-1}=$ $A_{p} T^{p}$, where $p=2.7-2.9$ and $A_{p}=10^{8} \mathrm{~s}^{-1} \mathrm{~K}^{-3}$. A similar relation $\tau_{e p}^{-1}=1.3 \times 10^{8} T^{3} \mathrm{~s}^{-1}$ is consistent with the data on temperature dependence of $\tau_{\varphi}$ obtained in Ref. 17 for Au films of $R_{\square} \simeq 7 \Omega$ in the temperature range $0.05-10 \mathrm{~K}$. Thus, for Au films $\tau_{\varphi}^{-1}$ and/or $\tau_{e p}^{-1}$ have a $T^{2}$ dependence in some cases and a $T^{3}$ dependence in other cases. The $\tau_{e p}^{-1} \propto T^{2}$ dependence was also found for $\mathrm{Nb}$ films from the effect of electron overheating in the temperature range 1.5-10K . The $\tau_{e p}^{-1} \propto T^{3}$ dependence was as a rule observed for pure films of other metals. 
For example, in Ref. 19 a temperature dependence of the inelastic time was obtained for pure $\mathrm{Al}$ films from weak localization. From this dependence the contribution of electronphonon scattering was separated, which agreed with $\tau_{e p}^{-1}=A_{p} T^{3}$ calculated from the real band structure of $\mathrm{Al}$ for electron scattering by longitudinal phonons. A similar result was also obtained for Al films later2 201. For pure $\mathrm{Cu}$ films the dependence $\tau_{e p}^{-1} \propto T^{3}$ for the time of electron-phonon energy relaxation at ultralow temperatures (10-300 $\mathrm{mK}$ ) was obtained from the effect of electron overheating 2 .

The effects of electron mean free path and film thickness on electron phase relaxation time in $\mathrm{Au}, \mathrm{Ag}$ and $\mathrm{Mg}$ films were studied in Ref. 13. The electron mean free path, $l$, was varied by annealing of quench-condensed films by stages. The experiments did not reveal any dependence of $\tau_{\varphi}$ on electron mean free path. The same result was obtained for the films of some other metals, for instance, $\mathrm{Sb}^{2} 3$ and $\mathrm{Bi} \mathrm{i}^{4}$. However, for $\mathrm{Nb}$ films the dependence upon the mean free path of the $\tau_{e p}^{-1} \propto l T^{2}$-type was found in Ref. 18. In our opinion, the results given in Refs. 23,24 may be attributed to a "pure limit". A contradictory situation appears in Ref. 18: the $\tau_{e p}(l)$ dependence points to the "dirty" limit, though the exponent on $\mathrm{T}$ is much below 4 .

This paper is concerned with the effect of disordering on characteristic electron relaxation times in thin Au films $\left(\tau_{\varphi}, \tau_{e p}, \tau_{s o}\right)$ estimated by analyzing the quantum corrections to conductivity. The disorder was enhanced by irradiating the films with $3.5 \mathrm{keV} \mathrm{Ar}$ ions, resulting in radiation damage of the crystal lattice. Variation in the fluence enabled us to prepare a series of samples of much the same thickness $(\simeq 10 \mathrm{~nm})$ with the values of $R_{\square}$ between 6 and $500 \Omega$. This permitted the previous data on manifestation of quantum interference effects in $\mathrm{Au}$ films 1217 to be complemented and new and, in some cases, unexpected features of disorder influence on phase and spin relaxation of electrons to be revealed.

\section{SAMPLES}

The Au films with thickness $\simeq 10 \mathrm{~nm}$ were prepared by condensing a metal molecular beam at a rate $0.05 \mathrm{~nm} \cdot \mathrm{s}^{-1}$ at pressure of residual gases $P=10^{-4} \mathrm{~Pa} .99 .99 \%$ purity gold was evaporated out of a Mo boat onto a sapphire single-crystal substrate (for measuring film conductivity) and a $\mathrm{NaCl}$ single-crystal cleft (for TEM study). Both substrates were precoated with a $\mathrm{SiO}$ sublayer $(\simeq 15 \mathrm{~nm}$ thick) to ensure identical structural and topological parameters of the deposited films. To enhance the structure disordering, the films were bombarded in vacuum with argon ions of energy $0.5-3.5 \mathrm{keV}$. The ion fluences $\Phi$ varied within $1 \times 10^{15}$ and $7 \times 10^{15} \mathrm{~cm}^{-2}$.

With this irradiation dose the decrease in the film thickness was negligible (10-20\% with the highest dose), but with a large dose holes with the sizes of $\leq 5 \mathrm{~nm}$ appeared in the film. The total surface fraction of the holes under the highest irradiation dose was $\leq 20 \%$. The electron microscopic results for the films are reported in Ref. 25.

As the irradiation dose was increased, the film resistance became tens of times higher against its initial value. We suggest that the irradiation induced increase in the film resistance is substantially associated with radiation defects generated in the lattice. For the interference effects under consideration, which result from frequently occurring elastic processes of electron scattering, the nature of scatterers is of no importance. What counts is that the electron motion be of diffusion nature because of small electron mean free paths. 
The effects of WL and EEI in the films studied manifested themselves in the dependences of resistance $R$ on perpendicular magnetic field $H$ (varied up to $20 \mathrm{kOe}$ ) and temperature (varied between 0.5 and $50 \mathrm{~K}$ ). The resistance measurements were carried out by using a standard four-probe de technique. The values of current $(5-20 \mu \mathrm{A})$ were chosen in such a way as to prevent, where possible, the influence of Joule heating. The relative error of the resistance measurements was $\leq 10^{-5}$. The film area under measurement was $2 \mathrm{~mm} \times$ $0.1 \mathrm{~mm}$ in size. The samples were places into a vacuum cryostat with a superconducting solenoid where the sapphire substrate was in a good thermal contact with a copper vessel filled with ${ }^{3} \mathrm{He}$ to produce temperatures below $1.5 \mathrm{~K}$.

Preliminary results on the problem discussed are reported in Ref. 25,26. Our earlier data and the new experimental results of this study were computer-processed using an advanced program to fit experiment results and theoretical formulas. A very good description was obtained, which made conclusions more reliable and convincing.

\section{CALCULATIONS}

To analyze the temperature and magnetic field dependences of conductivity $\sigma$, wellknown expressions for quantum corrections $\Delta \sigma(T, H)$ associated with WL and EEI effects in two-dimensional systems 10,1$]$ have been used. For the films studied, the two-dimensional conditions of manifestation of quantum interference effects are fulfilled: $L<L_{\varphi}, L_{T}$, where $L$ is the film thickness, $L_{\varphi}=\left(D \tau_{\varphi}\right)^{1 / 2}$ is the diffusion length of phase relaxation, $L_{T}=$ $(\hbar D / k T)^{1 / 2}$ is the thermal coherence length in a normal metal, and $D$ is the electron diffusion coefficient.

The contribution of WL and EEI to the temperature dependence of conductivity in a zero magnetic field is of the form:

$$
\Delta \sigma(T)=\frac{e^{2}}{2 \pi^{2} \hbar}\left\{-\left[\frac{3}{2} \ln \frac{\tau_{\varphi}^{*}}{\tau}-\frac{1}{2} \ln \frac{\tau_{\varphi}}{\tau}\right]+\lambda_{T}^{D} \ln \frac{k T \tau}{\hbar}\right\}
$$

where $\tau$ is the elastic electron relaxation time, $\tau_{\varphi}^{-1}=\tau_{\varphi 0}^{-1}+2 \tau_{s}^{-1} ;\left(\tau_{\varphi}^{*}\right)^{-1}=\tau_{\varphi 0}^{-1}+$ $(4 / 3) \tau_{s o}^{-1}+(2 / 3) \tau_{s}^{-1}, \tau_{\varphi 0}$ is the phase relaxation time due to inelastic scattering, $\lambda_{T}^{D}$ is the interaction constant in the diffusive channel. The latter can be written in terms of the universal constant $F$ - the angle-averaged amplitude of electron interaction with a small transferred momentum. $\lambda_{T}^{D}=1-(3 / 4) F$ in weak magnetic fields and $\lambda_{T}^{D}=1-(1 / 4) F$ in strong ones. For typical metals $F$ is close to zero. Below we use the presentation $\tau_{\varphi 0}^{-1} \propto T^{p}$, where $p$ is the exponent which depends on the mechanism of inelastic scattering. The first term in Eq. (3.1) corresponds to WL effects, while the second one to EEI. The variation of conductivity in an arbitrary temperature range from $T_{1}$ to $T_{2}$ due to WL and EEI effects is

$$
\sigma\left(T_{1}\right)-\sigma\left(T_{2}\right)=-\frac{e^{2}}{2 \pi^{2} \hbar}\left\{\left[\frac{3}{2} \ln \frac{\tau_{\varphi}^{*}\left(T_{1}\right)}{\tau_{\varphi}^{*}\left(T_{2}\right)}-\frac{1}{2} \ln \frac{\tau_{\varphi}\left(T_{1}\right)}{\tau_{\varphi}\left(T_{2}\right)}\right]+\lambda_{T}^{D} \ln \frac{T_{2}}{T_{1}}\right\}
$$

Below we use $-\Delta \sigma=(\Delta R) /\left(R R_{\square}\right)$, which is true for small corrections.

The variation of conductivity in a perpendicular magnetic field associated with the WL effect in two-dimensional system can be given by the expression: 


$$
\Delta \sigma(H)=\frac{e^{2}}{2 \pi^{2} \hbar}\left\{\frac{3}{2} f_{2}\left(\frac{4 e D H \tau_{\varphi}^{*}}{\hbar c}\right)-\frac{1}{2} f_{2}\left(\frac{4 e D H \tau_{\varphi}}{\hbar c}\right)\right\}
$$

where $f_{2}(x)=\ln (x)+\Psi(1 / 2+1 / x), \Psi$ is digamma function. For the films studied, the contribution of EEI to magnetoresistance (MR) is negligible, so we do not cite corresponding expressions.

\section{RESULTS AND DISCUSSION}

\section{A. $\Delta \sigma(T, H)$ dependences}

As an example, Fig. 1 1 shows the experimental $\Delta \sigma(H)$ dependences obtained at different temperatures (up to $50 \mathrm{~K}$ ) for one of the samples. It can be seen that the $\mathrm{MR}$ is positive which is the case of a strong spin-orbit interaction $\left(\tau_{s o} \ll \tau_{\varphi 0}(T)\right)$. However, the curves $\Delta \sigma(H)$ are nonmonotonic: for nonirradiated samples a well-defined maximum can be seen. The shape of $\Delta \sigma(H)$ curves reflects, according to Eq. (3.3), a competition of inelastic and spin-orbit electron scattering contributions.

Using computer fitting procedure according to Eq. (3.3) the characteristic values $D \tau_{\varphi}$ and $D \tau_{s o}$ can be found from the experimental data. In order to determine $\tau_{\varphi}$ and $\tau_{s o}$, the diffusion coefficient $D=(1 / 3) v_{F} l$ has been used, where Fermy velocity $v_{F}$ is found from the free-electron model and $l$ from the relation $\rho l=8.39 \times 10^{-12} \Omega \times \mathrm{cm}^{2}$ for Aut (where $\rho$ is the film resistivity, calculated, taking into account the electron-microscopic data for the topological structure of the films). The experimental magnetoresistive data are shown in Fig. 11 by dots, while solid curves represent the calculated $\Delta \sigma(H)$ dependences using Eq. (3.3) with $D \tau_{\varphi}$ and $D \tau_{s o}$ as fit parameters. As can be seen from Fig. 1 the fitting accuracy was high enough.

Fig. 2 shows a typical $D \tau_{\varphi}(T)$ dependence. The $D \tau_{\varphi}(T)$ dependences characterize the temperature variation of $\tau_{\varphi}$ since $\mathrm{D}$ may be assumed to be temperature-independent. It can be seen that the character of this dependence changes with decreasing temperature. Within the temperature range $10-50 \mathrm{~K}$, the $\tau_{\varphi} \propto T^{-p}$ dependence is revealed, where $p \approx 2$, in the range $3-10 \mathrm{~K}, \tau_{\varphi} \propto T^{-1}$ dependence has been observed for high-resistive samples and at lower temperatures $(T<2 \mathrm{~K}) D \tau_{\varphi}$ goes to some constant value.

The saturation of $\tau_{\varphi}(T)$ dependence at very low temperatures may be attributed to several factors. One of them is the influence of electron overheating caused by the measuring current. We however believe, that this factor is hardly probable since the used measuring currents were quite small. Besides, electron overheating does not lead to saturation and does not change the slope of the dependence $\tau_{\varphi}(T)$ though the $\tau_{\varphi}(T)$ values differ from the true ones. It is more likely that saturation of $\tau_{\varphi}(T)$ is due to spin electron scattering. In this case the saturation occures when spin scattering by magnetic impurities at very low temperatures starts predominating over the processes of electron-electron and electron-phonon scattering, and the characteristic time of spin scattering is temperature-independent.

A new explanation proposed in Ref. 27 attributes the low-temperature saturation of $\tau_{\varphi}(T)$ dependences in one-dimensional (1D) and two-dimensional (2D) electron systems to the influence of zero-point fluctuations of phase coherent electrons, which leads to intrinsic decoherence of electrons. This model accounts not only for its authors' results taken on 
narrow (quasi-1D) Au films, but it also explains the $\tau_{\varphi}(T)$ saturation in 1D and 2D metallic and semiconducting samples, observed by other reseachers. Besides, the characteristic saturation time $\tau_{0}$, calculated within the model of fluctuation decoherence, agrees well with the experiment. As the resistance of the samples increases (and hence the diffusion coefficient of the electrons decreases), the time $\tau_{0}$ becomes shorter. This regularity is observed, but only for high-ohmic samples with $R_{\square}>60 \Omega$, and the changes are considerably smaller than in Ref. 27. In our samples the saturation occures in comparatively low-Ohmic samples too. This implies that the model used in Ref. 27 cannot be the only explanation of saturation of $\tau_{\varphi}(T)$ dependence at low temperatures.

Above $4 \mathrm{~K}$, where the factors responsible for the low temperature saturation of the dependence $\tau_{\varphi}(T)$ turn out to be insignificant, this dependence can be approximated by

$$
\tau_{\varphi}^{-1}(T)=\tau_{e e}^{-1}(T)+\tau_{e p}^{-1}(T)
$$

in which the electron-electron relaxation rate is numerically determined by the expression in Ref. [10:

$$
\tau_{e e}^{-1}(T)=\frac{\pi k T}{\hbar} \frac{e^{2} R_{\square}}{2 \pi^{2} \hbar} \ln \left(\frac{\pi \hbar}{e^{2} R_{\square}}\right)
$$

Thus, at temperatures below $2 \mathrm{~K}$, the character of temperature dependence of $\tau_{\varphi}(T)$ depends on the times $\tau_{0}$ and $\tau_{s}$. At higher temperatures the shape of the curve $\tau_{\varphi}(T)$ is first dictated by the predominating electron-electron (ee) relaxation processes, and for the temperatures $T>20 \mathrm{~K}$ - by the processes of electron-phonon (ep) relaxation.

To separate the contributions of the e-e and e-p relaxation to $\tau_{\varphi}$, we used the following procedure. Assume that $\tau_{\varphi}^{-1}(T)$ is described by the expression

$$
\tau_{\varphi}^{-1}(T)=A T+A_{p} T^{p}
$$

or

$$
\left[\tau_{\varphi}(T)\right]^{-1}=A+A_{p} T^{p-1}
$$

It is not reliable to estimate the parameters $A, A_{p}$ and $p$ from the fitting of experimental results to Eq. (4.3), because the error is about $50 \%$ in this case. Our experimental dependences were plotted in the coordinates $1 /\left(\tau_{\varphi} T\right)$ vs. $T$. For high-Ohmic samples $\left(R_{\square}>60 \Omega\right)$, a horizontal part appears in the curve in the region of dominating electron-electron relaxation (see insert in Fig. 22) from which the coefficient A can be estimated. The corresponding $\tau_{e e}$ values turn out to be close to those, calculated from Eq. (4.2). It is also found in earlier studies Ref. 14,16 that the ratio of calculated and experimental $\tau_{e e}$-values for Au films is close to $1(\approx 1.05-1.2)$. This permits us to take $\tau_{e e}$ equal to the value, given by Eq. (4.2). Then, $A_{p}$, and $p$ can be found with good accuracy (the error is below 15\%).

For low-Ohmic samples $\left(R_{\square}<60 \Omega\right)$ the electron-electron relaxation is not essential since the inequality $\tau_{e e} \gg \tau_{e p}$ holds in the whole temperature range down to $\approx 5 \mathrm{~K}$. Spin-spin scattering or electron decoherence is observed below this temperature. For these samples no horizontal part is seen in the $1 /\left(\tau_{\varphi} T\right)$ vs. $T$ dependences and at $T>5 \mathrm{~K}$ the $\tau_{\varphi}(T)$ dependence actually characterizes electron-phonon relaxation. For low-Ohmic samples it can be described by the $\tau_{e p}^{-1} \propto T^{2}$ dependence. 
We have found that at enhancing disorder and growing $R_{\square}$ the $\tau_{\varphi}$ behavior is different in different temperature ranges: $\tau_{\varphi}$ increases in the region of predominating electron-phonon scattering and goes down in the region, where the e-e scattering makes itself evident. (Fig. (3)). The figure is a vivid demonstration of the general statement: as disorder increases, the electron-phonon interaction grows weaker, while electron-electron interaction is enhanced.

The reliability of obtained $\tau_{\varphi}$ and $\tau_{s o}$ values can be illustrated while comparing the temperature-resistance dependence calculated using $\tau_{\varphi}(T)$ and $\tau_{s o}$ derived from magnetoresistance with the experimental $R(T)$ data. The temperature variation of resistance in one of the samples is shown in Fig. T. At $T>20 \mathrm{~K}$ we observe the dependence $\Delta R / R \propto T^{2}$, which can be safely attributeded to electron-phonon-impurity interference 2 . (see also the experimental results in Ref. 17,29). At lower temperatures we see (Fig. (1) a horizontal part and then a weak growth of resistance as the temperature goes down. This behavior of the resistance can be explained well in terms of the WL and EEI effects. For the strong spin-orbit interaction $\left(\tau_{s o} \ll \tau_{\varphi 0}(T)\right.$ and $\left.\tau_{s} \gg \tau_{s o}, \tau_{\varphi 0}\right)$ Eq. (3.1) can be written as

$$
\frac{\Delta R}{R}=-a_{T} \frac{e^{2} R_{\square}}{2 \pi^{2} \hbar} \ln T+\text { const },
$$

where $a_{T}=1-p / 2$. For the electron-phonon interaction processes $p \approx 2$ and hence $a_{T} \approx 0$. If the electron-electron interaction predominates, $p=1$ and consequently $a_{T}=1 / 2$. Fig. . shows exactly this type of dependence $\Delta R(T)$. The open circles indicate the resistance calculated by Eq. (3.2) using $\tau_{\varphi}(T)$ and $\tau_{s o}$ found from magnetoresistance, while full circles represent the experimental $R(T)$ values. The agreement of calculations and the experiment is quite good.

Thus, the temperature behavior of quantum corrections $\Delta \sigma(T)$ for Au films studied is consistent with what is expected theoretically for $2 \mathrm{D}$ systems which are far-away from the percolation threshold. In the case of percolation effects, the coefficient $a_{T}$ has to be less than the theoretical value. Beside this, $\approx 1.5$-time decrease of $p$ in the expression $\tau_{\varphi}^{-1} \propto T^{p}$ is typical of percolation systems 5 . This effect has not been found in the films studied.

With the separated contribution of the e-e relaxation to $\tau_{\varphi}$ it turns out, that for highresistance samples $\left(R_{\square}>60 \Omega\right)$ the temperature dependence of $\tau_{e p}$ can be described by the function $\tau_{e p}^{-1} \propto A_{p} T^{p}$ where $p$ is slightly above 2 and increases monotonically with $R_{\square}$ (see the Table). The behavior of the immediate dependence of $\tau_{e p}$ on $l$ can be demonstrated if we plot the value of $\tau_{e p}$ versus $l$ for a certain temperature (Fig. 5, $T=20 \mathrm{~K}$ ). It turns out that some samples do not reveal any dependence of $\tau_{e p}$ on $l$ whereas the remaining samples feature a strong dependence of the form $\tau_{e p}^{-1} \propto l$.

The increase in the exponent $p$ with enhancing disorder (see the Table) and the existence of the above-mentioned relationship between $\tau_{e p}$ and $l\left(\tau_{e p}^{-1} \propto l\right)$ suggest that as the films are disordered, there occurs a pure $\left(q_{T} l>1\right)$ - dirty $\left(q_{T} l<1\right)$ limit transition. Indeed, the condition $q_{T} l \simeq 1$ defines a particular temperature $T_{t r}=\hbar s_{l, t} / k l\left(s_{l, t}\right.$ is the velocity of phonons of different polarization) below which a dirty limit case occurs, and this temperature increases with decreasing $l$ (see the Table). In those cases where $T_{t r}$ is below the temperature range within which the dependence $\tau_{\varphi}^{-1} \propto T^{2}$ makes itself evident, i.e. $T_{t r}<10 \mathrm{~K}$, there is no influence of electron mean free path on $\tau_{e p}$. However when $T_{t r}$ is much higher than $10 \mathrm{~K}$, an increase in $p$ is observed and the relation between $\tau_{e p}$ and $l$ appears.

In view of the above behavior, the discrepancy between the $p$-values for $\mathrm{Au}$ films given in Refs. 12 17 can be explained. The subjects of investigation in Refs. 12,13,15 correspond 
to a pure limit case, where $p$ is found to be equal to 2 ; the data in Ref. 16 are true for a dirty limit case $(0.03-1.0 \mathrm{~K})$ where $p$ took a value close to 3 .

The problem of reduction of the exponent $p$ as opposed to the theoretical predictions

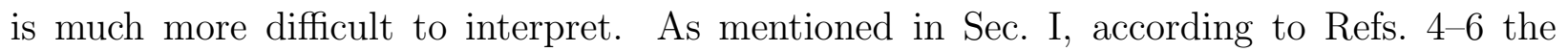
bulk disordered samples must reveal dependences $\tau_{e p}^{-1} \propto l T^{4}$ for $q_{T} l<1$ and $\tau_{e p}^{-1} \propto T^{3}$ for $q_{T} l>1$. Since $\tau_{e p}$ is given by the Eliashberg function $\alpha^{2}(\omega) F(\omega)$ within the frequency range corresponding to the energy of thermal phonons30.

$$
\tau_{e p}^{-1}=4 \pi \int d \omega \frac{\alpha^{2}(\omega) F(\omega)}{\sinh (\hbar \omega / k T)},
$$

the reduction of $p$ from 3 to 2 within the pure limit corresponds to the occurrence of a linear, rather than quadratic, dependence of the Eliashberg function on $\omega$ at low frequencies.

The change in the Eliashberg function form at low phonon frequencies may be accounted for by the modification of the phonon spectrum in thin films. In this case for the 2D phonon spectrum a dependence $\tau_{e p}^{-1} \propto l T^{3}$ is assumed to be observed in the dirty limit 31.32 and $\tau_{e p}^{-1} \propto T^{2}$ in the pure one (rigorous theory is unavailable). Assuming two-dimensionality of the phonon spectrum allowed the authors of Ref. 16 to fit the calculated data on $\tau_{e p}$ with the experimental ones, and the process of heat escape to the environment at electron overheating was successfully interpreted in Ref. 33 on the same ground.

Many authors believe that the 2D phonon spectrum in a thin film is due to the quantum size effect for the phonons when the phonon wavelength $\lambda=2 \pi / q$ is comparable to the film thickness. This problem is consequently discussed for free standing film. For $L<\lambda$ there exist flexural waves with the quadratic dispersion relations $\omega \propto q^{2}$ (Ref. 34) in a free standing film which are characterized by a linear dependence of phonon state density on $\omega$ : $F(\omega) \propto \omega$. Since $q_{T}=2 k T / \hbar s_{l, t}$, for Au films $10 \mathrm{~nm}$ thick the above condition is realized at temperatures below $3 \mathrm{~K}$. At higher temperatures the phonon spectrum of free standing film is also quantized 35 . Thus, one can expect a $2 \mathrm{D}$ behavior of phonons due to the size quantization in a free standing film or in a film with weak adhesion to the substrate. For the film on substrate only those phonons are prone to quantization which have undergone complete internal reflection from the metal-substrate boundary, i.e. fly up to the boundary at an angle larger than the critical one $\theta_{c r}\left(\sin \theta_{c r}=s_{l, t} / s_{l, t}^{*}\right.$, the angle is measured from the normal to the boundary and $*$ refers to the substrate). For the considered gold-sapphire system $\theta_{c r}^{l}=17^{\circ}$ and $\theta_{c r}^{t}=10^{\circ}$. The phonons within the critical angle accomplish acoustic metal-substrate coupling.

We should bear in mind that in the studied real film-substrate systems the acoustic coupling is far from being weak. Therefore, the results for free films cannot be applied in our case in full measure.

The interpretation of the problem proposed in Ref. 36 takes into account the fact that the film is on a substrate. Concurrent with the above mentioned waves, we suppose that Love's waves may occur in the film-substrate system. The Love's waves are surface waves whose displacement vector is parallel to the surface and perpendicular to the direction of propagation. Solution of the problem for the Love's waves in a film-substrate system results in an uncommon dispersion relation. Thus, when the conditions $s_{l, t} \ll s_{l, t}^{*}$ and $d^{*} \ll d(d$ being the medium density) are met, the dispersion relation for the Love's waves is of the form $\omega \propto q_{T}^{1 / 2}$ (Ref. 37) similar to that for 2D plasmons. These conditions are well fulfilled for 
the gold-sapphire system. The 1D Love's wave which obeyed the above dispersion relation, gives rise to a linear frequency spectrum 36 , which is possibly responsible for the observed behavior of the temperature dependence of $\tau_{e p}^{-1}$ for the gold films on sapphire substrates.

\section{B. The effect of disordering on $\tau_{s o}$ in thin films}

Now we discuss the behavior of $\tau_{\text {so }}$ on disordering in the temperature region where $\tau_{s} \gg \tau_{\varphi 0}, \tau_{s o}$. The values of $\tau_{s o}$ was found to increase with disorder and the increase in $R_{\square}$ (see the Table). This result is quite unexpected because it is commonly assumed that the increase in the frequency of elastic scattering processes $\tau^{-1}$ also involves an increase in the frequency of spin-orbit processes $\tau_{s o}^{-1}$. The ratio of the above frequencies, $\tau_{s o}^{-1} / \tau^{-1}$, characterizes the probability of spin-orbit process on elastic electron scattering. An approximate estimation of this quantity can be given by the following relationship for metals with inversion symmetry of crystal lattice 38 :

$$
\tau_{s o}^{-1} / \tau^{-1} \sim\left(\alpha Z_{i}\right)^{4}
$$

if the spin-orbit interaction is of importance in the field of a heavy impurity, or

$$
\tau_{s o}^{-1} / \tau^{-1} \sim(\alpha Z)^{4}
$$

if the spin-orbit interaction is essential in the matrix (on scattering by a light impurity). Here $\alpha=e^{2} / \hbar c \simeq 1 / 137$ is the fine structure constant, $Z_{i}$ and $Z$ are the atomic numbers of the impurity and the matrix (host metal), respectively.

The dependence of $\tau_{s o}^{-1} / \tau^{-1}$ on $Z$ defined by Eq. (4.8) was verified for films of ten different metals in Ref. 39. The verification was made for $\tau_{\text {so }}$ determined from the Knight shift, critical magnetic field and tunnel conductivity of superconductors. The time of electron flight from surface to surface, $\tau^{s f}=L / v_{F}$, that is the time between two acts of surface scattering, was used as $\tau$. The dependence of $\tau^{s f} / \tau_{s o}$ on $Z$ was truly close to $Z^{4}$, and the values of $\epsilon=\tau^{s f} / \tau_{\text {so }}$ corresponded to the probability of surface scattering followed by a spin flip appeared to be rather high $\left(5 \times 10^{-1}-5 \times 10^{-3}\right)$ for heavy metals $(\mathrm{Pb}, \mathrm{Sn}, \mathrm{Ga}, \mathrm{Cu})$ compared to the expected values for bulk scattering (approximately by a factor of three). In Refs. 40,41 the values of $\tau_{s o}$ for different degrees of disordering were determined from the effects of weak localization in $\mathrm{Mg}, \mathrm{Ag}$ and $\mathrm{Al}$ films and the validity of the relation $\tau_{s o}^{-1} / \tau^{-1}=\epsilon=$ const was confirmed.

One would think that the uncommon behavior of $\tau_{\text {so }}$ with disordering, observed by us in the Au films, could be attributed to the modification of the nature of main scatterers in the film after irradiation with Ar ions: the number of scatterers and, hence, the frequency of elastic scattering processes, $\tau^{-1}$, after the irradiation increases while the proportion of scatterers with strong spin-orbit interaction decreases. This could have been due to the implanted Ar ions if the latter had a weak spin-orbit interaction compared to the matrix atoms. However, it seems to us that the high concentration of Ar ions in the film is unlikely because for their average energy of $2 \mathrm{keV}$ their free path within the irradiated film is more than the film thickness (a significant part of ions with high energy pierces the film and penetrates the $\mathrm{SiO}$ sublayer). Moreover, the film heating on irradiation (up to $\simeq 500$ $600^{\circ} \mathrm{C}$ ) results in that a large part of the implanted Ar ions escapes it. By the data given in Ref. 42, the maximum Ar concentration in irradiated films of noble metals is no more than 
1\%. For significant influence of Ar impurities on $\tau_{s o}$, it is necessary that the Ar concentration should be higher at least by the order of magnitude.

Let us assume that the Ar irradiation results in an increase in the concentration of radiation defects of the film structure and leads to enhancement of diffusion behavior of electron motion in the film. If the dominant mechanism of elastic scattering in the perfect film is a surface scattering, it turns out that the enhancement of disordering causes a bulk scattering to be dominant. As in Ref. 13, the frequency $\tau_{s o}$ can be represented as a sum of contributions from bulk and surface scattering to spin-orbit relaxation:

$$
\frac{1}{\tau_{s o}}=\frac{1}{\tau_{s o}^{b}}+\frac{1}{\tau_{s o}^{s f}}=\frac{\epsilon^{b}}{\tau_{b}}+\frac{\epsilon^{s f}}{\tau^{s f}} .
$$

It is quite possible that the probability of spin flip under electron scattering by surface is much higher than under scattering by impurity in the bulk, i.e. $\epsilon^{s f} \gg \epsilon^{b}$. This suggestion is supported by the experimental results presented in Ref. 13, where the dependence of $\tau_{s o}^{-1}$ on $\mathrm{Mg}$ film thickness was studied. The same result was obtained for films of different metals in Ref. 39 and also for Bi films in Ref. 14 where the localization correction to magnetoresistance was studied in parallel magnetic field in conditions where the quantum size effect manifests itself.

In view of the above inequality, the processes of spin-orbit scattering by surface are to be dominant in perfect films where the second term prevails. With enhancing disorder, the contribution of these processes decreases because of the increase in the time, $\tau^{s f}$, of diffusion electron motion from surface to surface. The contribution of spin-orbit scattering by impurities in the bulk material having the probability $\epsilon^{b} \ll \epsilon^{s f}$ becomes appreciable. This increases the time $\tau_{s o}$. We can illustrate this by a simple transformation of Eq. (4.9). Let us assume, that $\tau^{b} \approx l / v_{F}$, and the time of diffusion from one surface to the other is $\tau^{s f} \approx L^{2} / D$, and $D \approx v_{F} l$. Then Eq. (4.9) becomes

$$
\frac{1}{\tau_{s o}}=\frac{\epsilon^{b} v_{F}}{l}+\frac{\epsilon^{s f} v_{F} l}{L^{2}}
$$

or

$$
\frac{l}{v_{F}} \frac{1}{\tau_{s o}}=\epsilon^{b}+\epsilon^{s f} \frac{l^{2}}{L^{2}}
$$

The experimental points are shown in Fig. 6 in the $\left(l / v_{F}\right) / \tau_{s o}$ vs. $l^{2}$ coordinates. The straight line in Fig. 6 obtained by the least-square technique has $\epsilon^{b}=4 \times 10^{-4}$ and $\epsilon^{s f}=$ $2 \times 10^{-2}$, which supports the assumption that $\epsilon^{s f} \gg \epsilon^{b}$. Thus, with the high probability of spin flip during the electron scattering at the surface, the enhanced disorder in the film reduces the frequency of collisions with the surface and is responsible for the decrease in the spin-orbit scattering rate, observed with decreasing of electron mean free path.

\section{CONCLUSIONS}

The irradiation of $\mathrm{Au}$ films with $3.5 \mathrm{keV} \mathrm{Ar}$ ions has made it possible to obtain samples with the crystal lattice disorder in a wide range. The experiments resulted in the previously unknown influence of disorder on the processes of phase and spin-orbit relaxation of electrons. 
1. It is clearly demonstrated that progressing disorder enhances the electron-electron scattering and weakens the the electron-phonon scattering (Fig. (1).

2. For a particular degree of disordering, the frequency of electron-phonon scattering begins to decrease directly with the electron mean free path and the exponent $p$ in temperature dependence $\tau_{e p}^{-1} \propto T^{p}$ somewhat increases compared to $p=2$ for weakly disordered films (see the Table). The variation in $\tau_{e p}$ on disordering may be attributed to the pure-dirty limit transition.

3. Attention is attracted to the fact that the reduced values of exponents $p$ in the "pure" limits (cf. the theoretical predictions for 3D metals) are related to the surface waves of Lovetype, which present in the film-substrate system. These waves obey an unusual dispersion law and have a linear frequency spectrum.

4. The abnormal decrease in the rate of spin-orbit scattering with enhancing disorder is treated assuming that the probability of spin flip is higher under electron scattering by surface than by impurities in the bulk.

\section{ACKNOWLEDGMENTS}

We are very grateful to V.V.Bobkov and V.I.Glushko for Ar-ion irradiation of Au films and electron microscopy study, A.I.Kopeliovich, V.A.Shklovsky and E.S.Syrkin for their

helpful discussion of the results and E.Yu.Kopeichenko for his assistance in the processing of the experimental data. Our research work has been partially supported by the International Science Foundation (grant U2G000). 


\section{REFERENCES}

* E-mail: belevtsev@ilt.kharkov.ua

${ }^{1}$ B.L.Altshuler and A.G.Aronov in Modern Problems in Condensed Matter Science, edited by A. L. Efros and M. P. Pollak (North-Holland Publ. Co, Amsterdam, 1985), p.1

${ }^{2}$ H.Fukuyama in Modern Problems in Condensed Matter Science, edited by A. L. Efros and M. P. Pollak (North-Holland Publ. Co, Amsterdam, 1985), p.155.

${ }^{3}$ M. Yu. Reiser, Phys. Rev. B40, 5411 (1989).

${ }^{4}$ J. Rammer and A. Schmid, Phys. Rev. B34, 1352 (1986).

${ }^{5}$ A. Schmid, Z. Phys. 259, 421 (1973).

${ }^{6}$ M. Yu. Reizer and A. V. Sergeev, Zh. Eksp. Teor. Fiz. 90, 1056 (1986) [Sov. Phys. - JETP 63, 616 (1986)].

${ }^{7}$ H. Takayama, Z. Phys. 263, 323 (1973).

${ }^{8}$ E. Abrahams, P. W. Anderson, D. C. Licciardello, and T. V. Ramakrishnan, Phys. Rev. Lett. 42, 673 (1979).

${ }^{9}$ P. A. Lee and T. V. Ramakrishnan, Rev. Mod. Phys. 53, 287 (1985).

${ }^{10}$ B. L. Altshuler, A. G. Aronov, M. E. Gershenson, and Yu. V. Sharvin, Sov. Sci. Rev. A9, (Harwood Acad. Publ. GmbH, Schur (Switzerland), 1987), p.223.

${ }^{11}$ G. Bergmann, Phys. Rep. 107, 1 (1984).

${ }^{12}$ G. Bergmann, Z.Phys. B48, 5 (1982).

${ }^{13}$ R. P. Peters and G. Bergmann, J. Phys. Soc. Jap. 54, 3478 (1985).

${ }^{14}$ G. Bergmann, Wei Wei, Yao Zou, and R. M. Mueller, Phys.Rev. B41, 7386 (1990).

${ }^{15}$ G. Dumpich and A. Carl, Phys. Rev. B43, 12074 (1991).

${ }^{16}$ P. M. Echternach, R. M. Thoman, C. M. Gould, and H. M. Bozler, Phys. Rev. B46, 10339 (1992).

${ }^{17}$ P. M. Echternach, M. E. Gershenson, and H. M. Bozler, Phys. Rev. B47, 13659 (1993).

${ }^{18}$ E. M. Gershenzon, M. E. Gershenzon, G. N. Goltsman, A. Yu. Lyulkin, A. D. Semenov, and A.V.Sergeev, Zh. Eksp. Teor. Fiz. 97, 901 (1990) [Sov. Phys. - JETP 70, 505 (1990)].

${ }^{19}$ P. Santanam and D. E. Prober, Phys. Rev. B29, 3733 (1983).

${ }^{20}$ J. M.Gordon and A. M. Goldman, Phys. Rev. B34, 1500 (1986).

${ }^{21}$ Y. K. Kwong, K. Lin, M. S. Isaacson and J. M. Parpia, J. Low Temp. Phys. 88, 261 (1992).

${ }^{22}$ M. L. Roukes, M. R. Freeman, R.S.Germain, R. C. Richardson and M. B. Ketchen, Phys. Rev. Lett. 55, 422 (1985).

${ }^{23}$ J. Liu and N. Giordano, Phys. Rev. B43, 3928 (1991).

${ }^{24}$ Yu. F. Komnik, V. Yu. Kashirin, B. I. Belevtsev and E. Yu. Beliayev, Fiz. Nizk. Temp. 20, 158 (1994) [Low Temp. Phys. 20, 127 (1994)].

${ }^{25}$ B. I. Belevtsev, E. Yu. Beliayev, V. V. Bobkov and V. I. Glushko, Fiz. Nizk. Temp. 21, 763 (1995) [Low Temp. Phys. 21, 592 (1995)].

${ }^{26}$ B. I. Belevtsev, Yu. F. Komnik, and E. Yu. Beliayev, Fiz. Nizk. Temp. 21, 839 (1995) [Low Temp. Phys. 21, 646 (1995)].

${ }^{27}$ P. Mohanty, E. M. Q. Jariwala, and R. A. Webb, Phys.Rev.Lett. 78, 3366 (1997)

${ }^{28}$ M. Yu. Reizer and A. V. Sergeev, Zh. Eksp. Teor. Fiz. 92, 2291 (1987) [Sov. Phys. - JETP 65, 1291 (1987)].

${ }^{29}$ N. G. Ptitsina, C. M. Chulkova, K. S. Il'in, A. V. Sergeev, F. S. Pochinkov, E. M. Gershenzon, M. E. Gershenzon Phys. Rev. B56, 10089 (1997). 
${ }^{30}$ B. Keck and A. Schmid, J. Low Temp. Phys. 24, 611 (1976).

${ }^{31}$ D. Belitz and S. Das Sarma, Phys. Rev. B36, 7701 (1987).

${ }^{32}$ V. A. Shklovskii, Fiz. Tverd. Tela (St.Petersburg) 34, 2844 (1992) [Sov. Phys. - Solid State 34, 1520 (1992)].

33 J. C. Nabity and M. N. Wybourne, Phys. Rev. B44, 8990 (1988).

${ }^{34}$ I. M. Lifshits, Zh. Eksp. Teor. Fiz 22, 471 (1952).

${ }^{35}$ B. A. Tavger and V. Ya. Demikhovskiy, Izv. Vyssh. Uchebn. Zaved., ser. Fiz., No 4, 130 (1966).

${ }^{36}$ E. S. Syrkin, Yu. F. Komnik and E. Yu. Beliayev, Fiz. Nizk. Temp. 22, 107 (1996) [Low Temp. Phys. 22, 80 (1996)].

${ }^{37}$ Yu. A. Kosevich and E. S. Syrkin, Akust. Zh. 34, 113 (1988) [Sov. Phys. - Acoust. 34, 61 (1988)].

${ }^{38}$ A. A. Abrikosov and L. P. Gor'kov, Zh. Eksp. Teor. Fiz. 42, 1088 (1962) [Sov.Phys. JETP 15, 752, (1962)].

${ }^{39}$ R. Meservey and P. M. Tedrow, Phys. Rev. Lett. 41, 805 (1978).

${ }^{40}$ G. Bergmann and C. Horriar-Esser, Phys. Rev. B31, 1161 (1985).

${ }^{41}$ P. Santhanam, S. Wild, and D. E. Prober, Phys. Rev. B35, 3188 (1987).

${ }^{42}$ F. Parmigiani, E. Kay, T. C. Huang, J. Perrin, M. Jurich, and J. D. Swallen, Phys. Rev. B33, 879 (1986).

${ }^{43}$ P. E. Lindelof and Shiguang Wang, Phys. Rev. B33, 1478 (1986).

${ }^{44}$ A. V. Butenko, Yu. F. Komnik and E. I. Bukhshtab, Fiz. Nizk. Temp. 9, 1171 (1983) [Sov. J. Low Temp. Phys. 9, 52 (1983)]. 


\section{FIGURES}

FIG. 1. Quantum correction to Au film conductivity versus perpendicular magnetic field for the sample with $R_{\square}=14.7 \Omega$ at different temperatures. From top to bottom $T=0.56,4.5,8,16$,

$25 \mathrm{~K}$. The circles are experimental results and solid lines are the theoretically calculated quantum corrections for corresponding temperatures.

FIG. 2. Temperature dependences of $D \tau_{\varphi}$ for the samples with $R_{\square} \simeq 437 \Omega$ (1) and $R_{\square} \simeq 85 \Omega$ (2). Insert: dependence of $1 / \tau_{\varphi} T$ on $T ;-\cdots \tau_{e e}$, calculated by Eq.(4.2)

FIG. 3. Dependence of electron phase relaxation time upon $R_{\square}$ at various temperatures T, K: $\bigcirc-4, \square-5, \triangle-20, \diamond-30$. The dashed line is only a guide to the eye.

FIG. 4. Temperature dependence of Au film resistance. Filled circles correspond to experimental data, while open ones represent theoretical values found from Eq.(3.2), using the $\tau_{\varphi}$ values calculated from quantum corrections to conductivity in magnetic field.

FIG. 5. The electron-phonon relaxation time versus electron mean free path for different $\mathrm{Au}$ films at $T=20 \mathrm{~K}$. The dashed line is only a guide to the eye.

FIG. 6. Fitting the experimental results by Eq.(4.11). 
Table

\begin{tabular}{|l|l|l|l|}
\hline \multicolumn{1}{|c|}{$R_{\square}(\Omega)$} & \multicolumn{1}{|c|}{$T_{t r}(K)$} & \multicolumn{1}{c|}{$\tau_{s o}(\mathrm{~s})$} \\
\hline 10.6 & 3.0 & $2.0_{-}^{+} 0.1$ & $3.8 \times 10^{-13}$ \\
14.6 & 4.3 & $2.0_{-}^{+} 0.1$ & $5.3 \times 10^{-13}$ \\
14.7 & 4.3 & $2.0_{-}^{+} 0.1$ & $6.2 \times 10^{-13}$ \\
20.4 & 6.0 & $2.0_{-}^{+} 0.1$ & $5 \times 10^{-13}$ \\
44.4 & 11 & $1.9_{-}^{+} 0.1$ & $8.5 \times 10^{-13}$ \\
61.4 & 18 & $2.0_{-}^{+} 0.1$ & $1 \times 10^{-12}$ \\
85 & 25 & $2.2_{-}^{+} 0.1$ & $3 \times 10^{-12}$ \\
143 & 37 & $2.3_{-}^{+} 0.1$ & $4.8 \times 10^{-12}$ \\
161 & 43 & $2.55_{-}^{+} 0.1$ & $6.1 \times 10^{-12}$ \\
437 & 103 & $2.8_{-}^{+} 0.15$ & $1.2 \times 10^{-11}$ \\
\hline
\end{tabular}




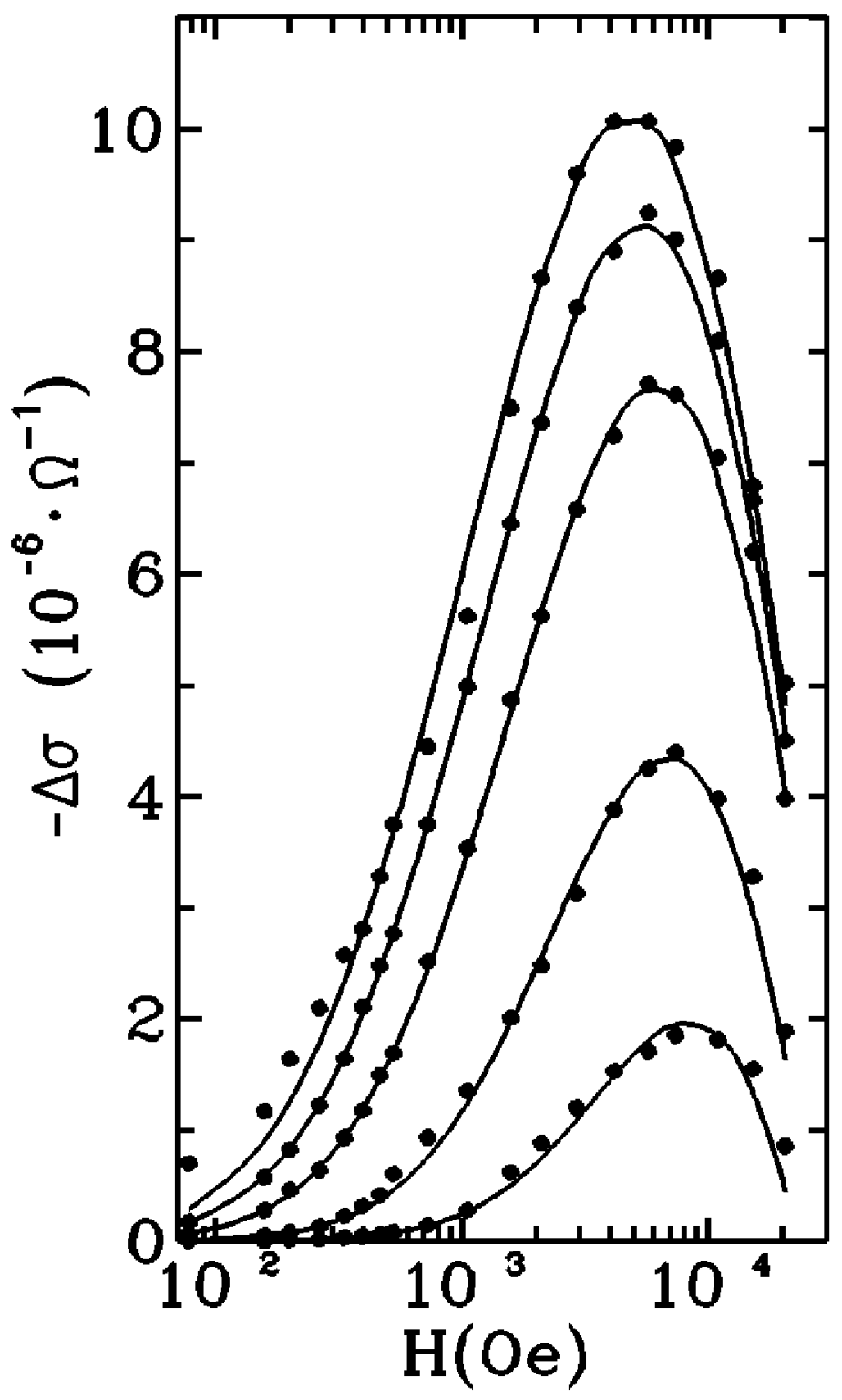

Fig.1 to paper Belevtsev B. I. et al. 


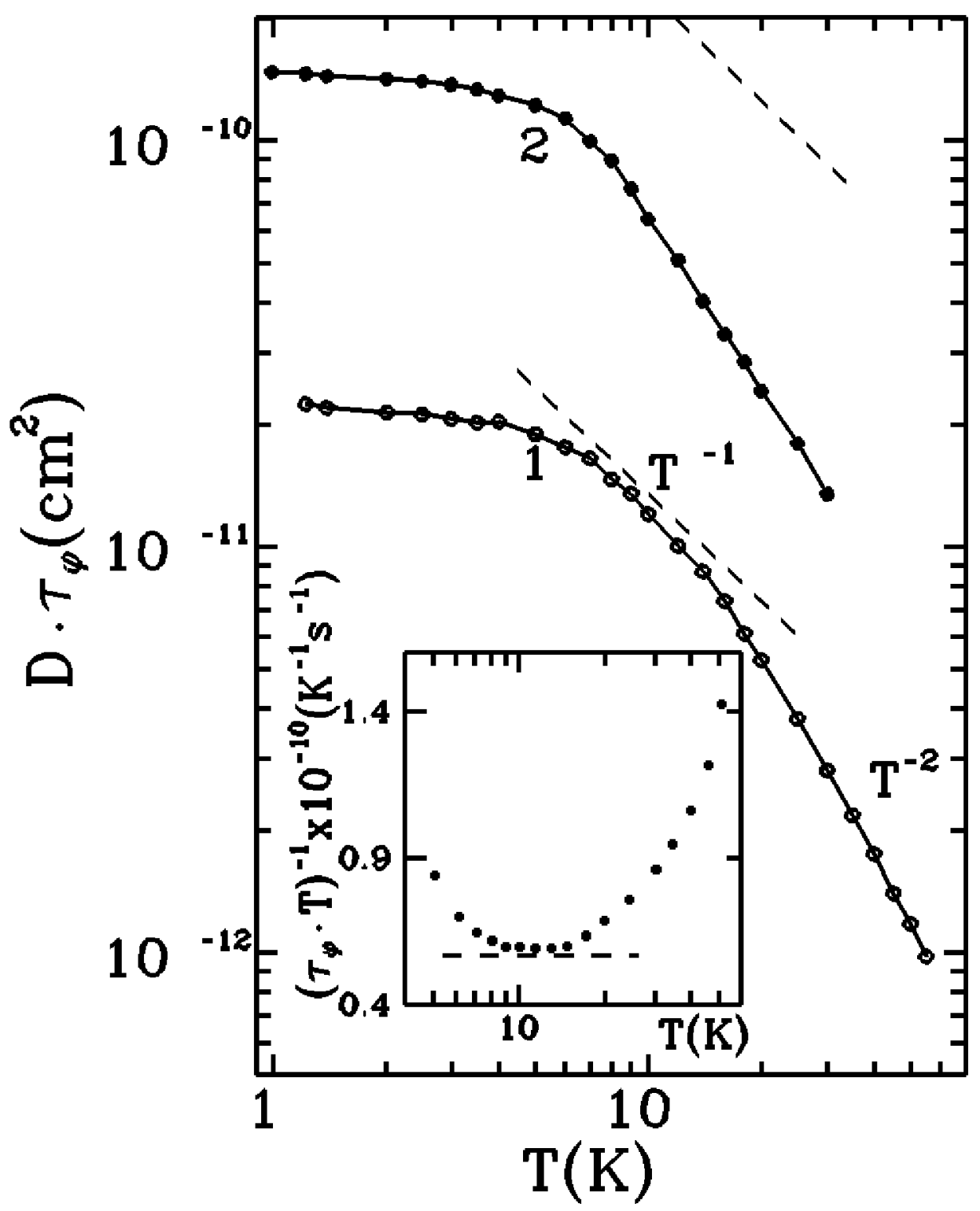

Fig.2 to paper Belevtsev B. I. et al. 


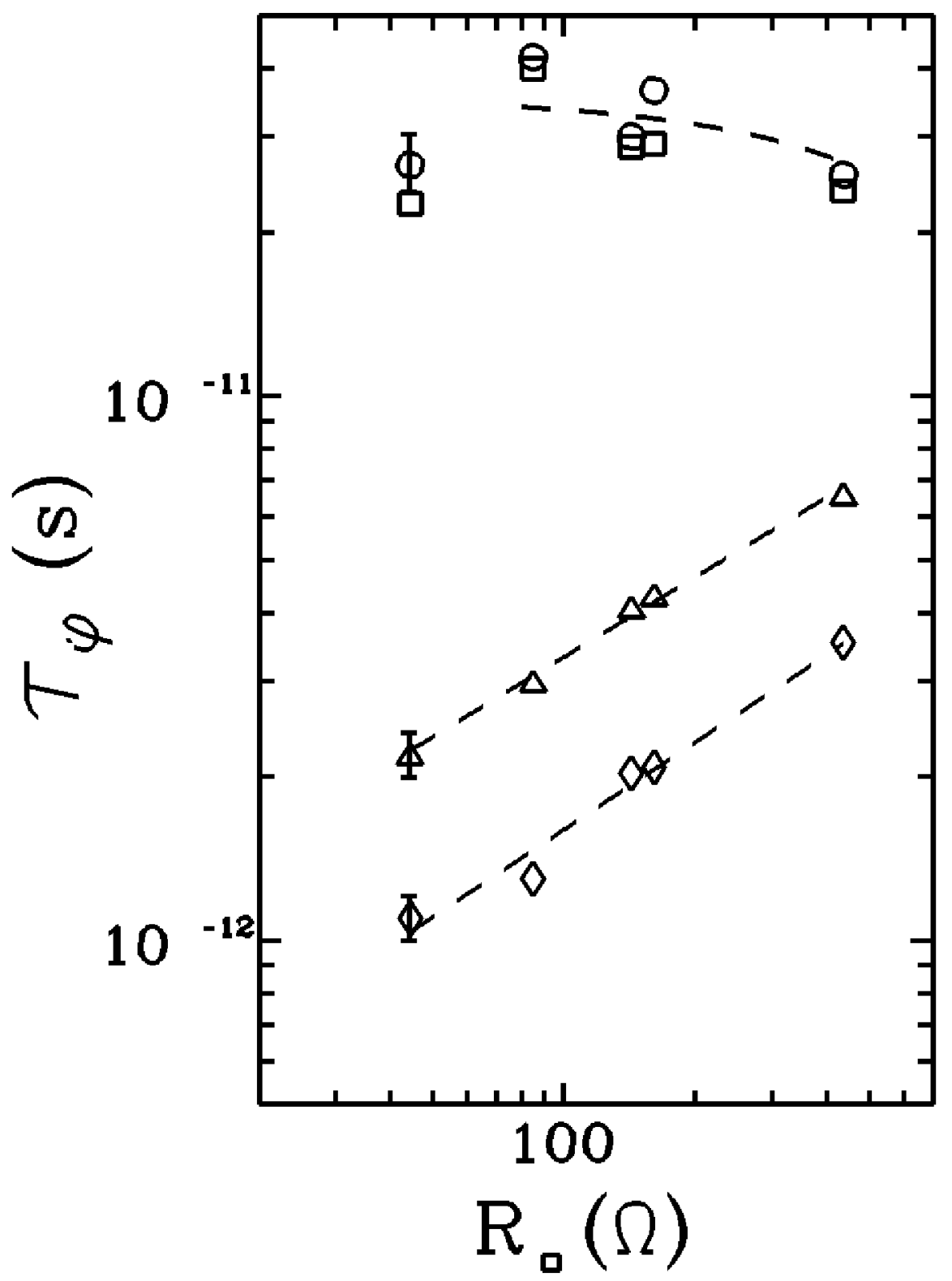

Fig.3 to paper Belevtsev B. I. et al. 


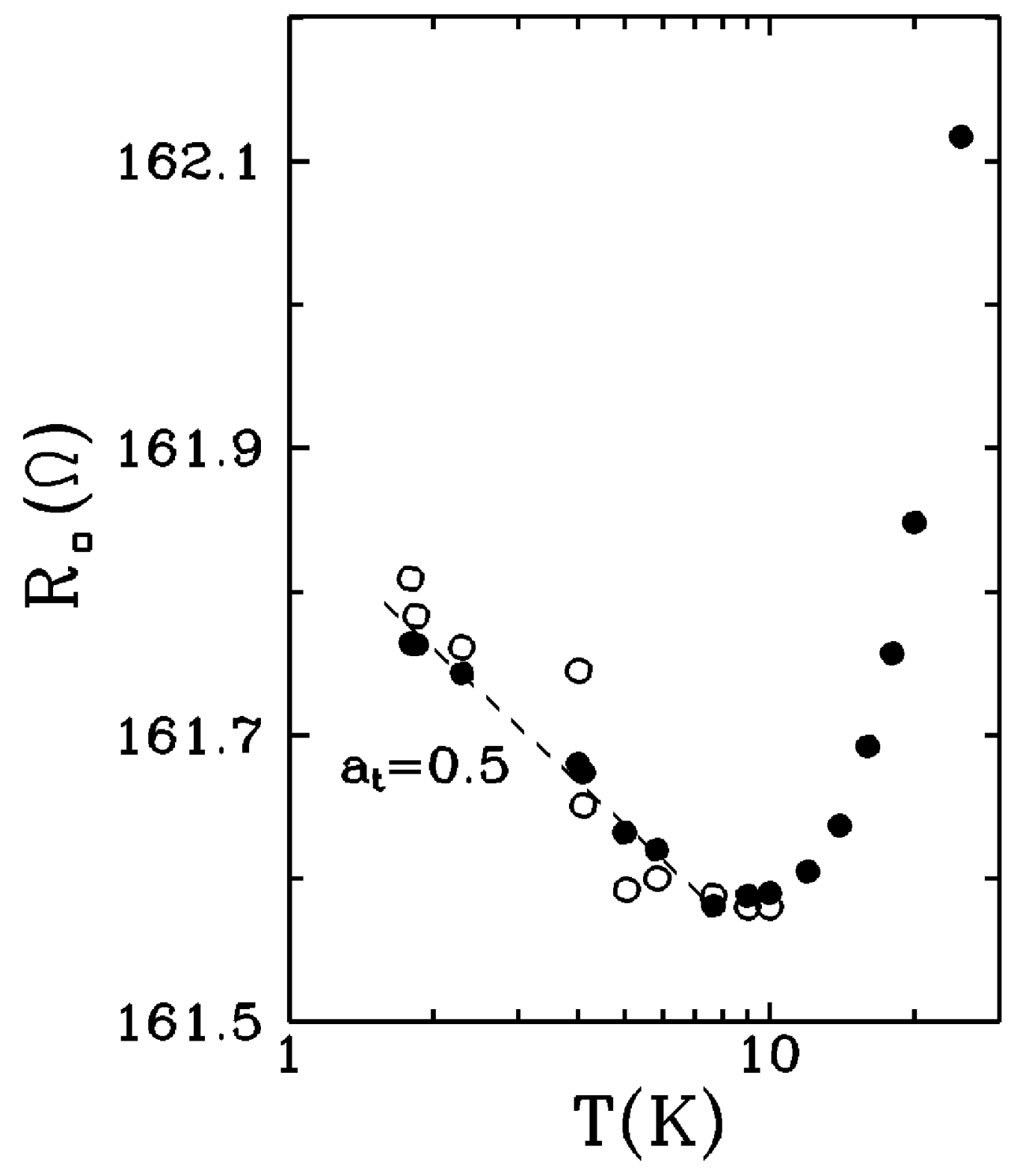

Fig.4 to paper Belevtsev B. I. et al. 


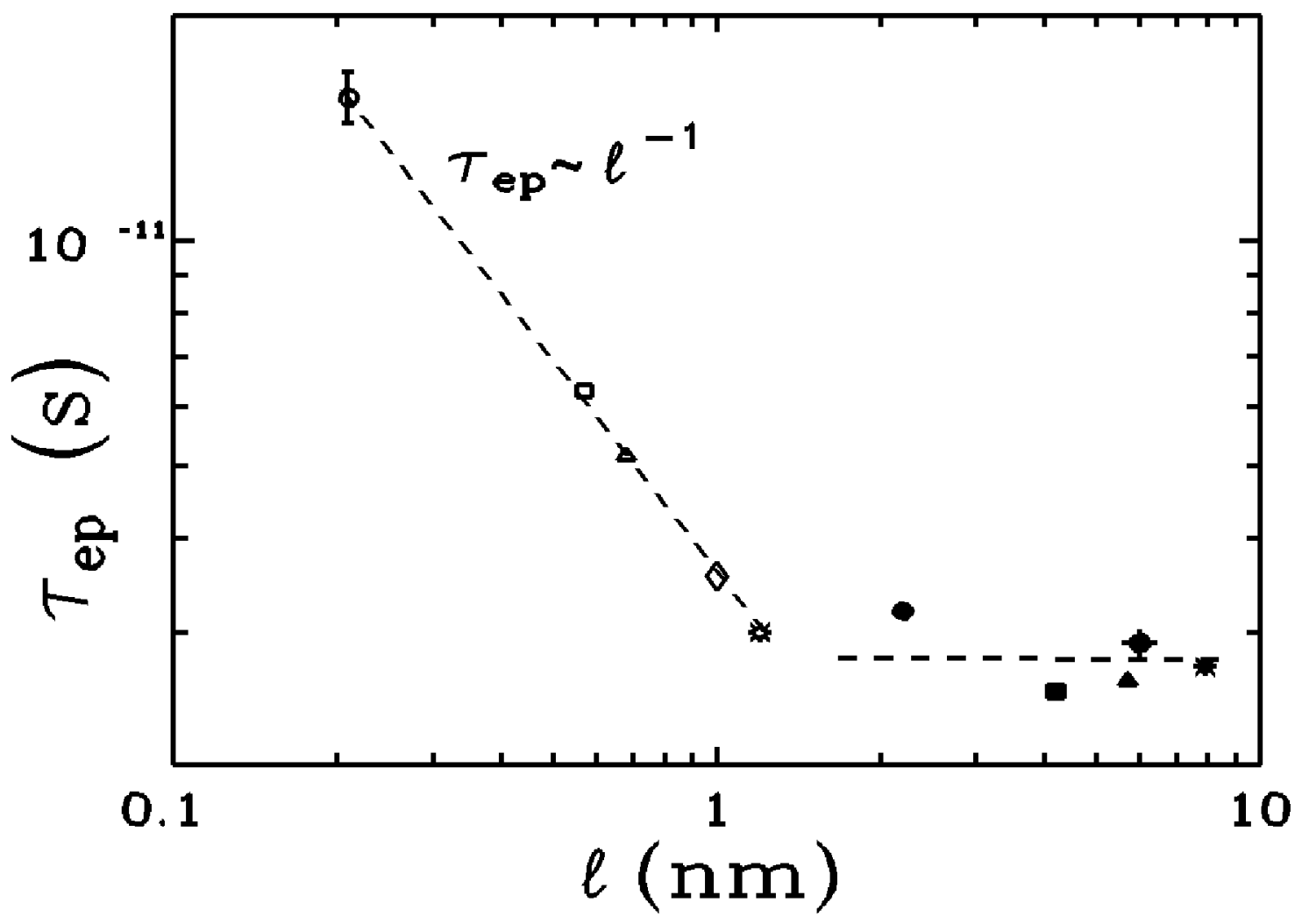

Fig.5 to paper Belevtsev B. I. et al. 


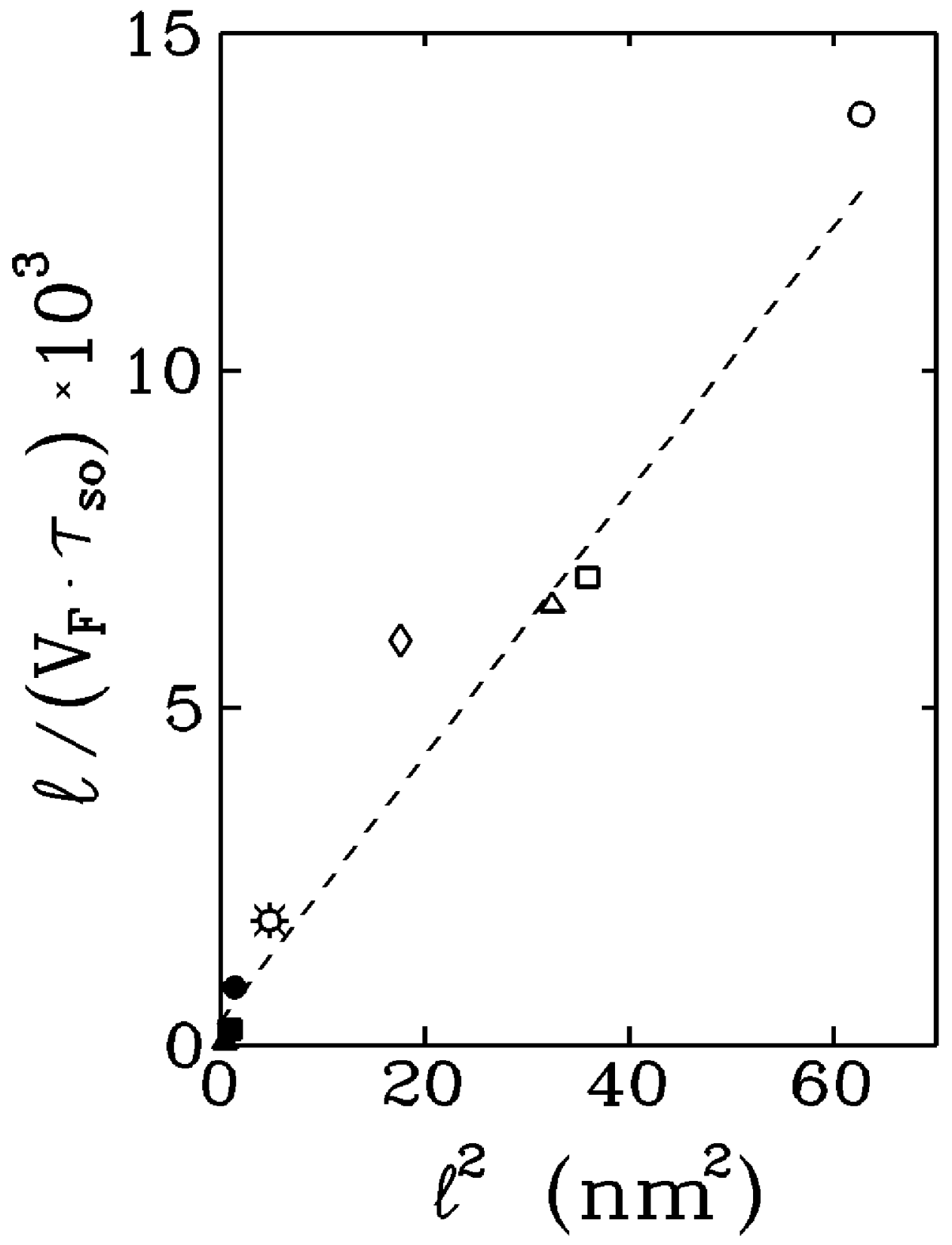

Fig.6 to paper Belevtsev B. I. et al. 